\title{
Microenvironnement immunitaire et cancer
}

> L'histoire naturelle du cancer implique des interactions entre la tumeur et le système immunitaire de l'hôte. Chez l'homme, un faisceau d'arguments cliniques et expérimentaux attestent de l'existence d'une immunité naturelle antitumorale. Dans les cancers colorectaux, nous avons observé un impact pronostic majeur de l'infiltration immunitaire, indépendant de la classification TNM (T, tumeur primitive; $N$, ganglions lymphatiques [nodes]; $M$, métastase). Un score immunitaire adapté à la pratique clinique a été créé. Ce score, dénommé immunoscore, fait actuellement l'objet d'une validation internationale pour les cancers du côlon. L'utilisation de l'immunoscore dans la pratique clinique pourrait améliorer l'appréciation pronostique et la prise en charge thérapeutique des patients. <

Le concept d'immunosurveillance, énoncé en 1957 par Sir Mac-Farlane Burnet et Lewis Thomas [1], constitue le fondement de l'immunocancérologie. II stipule que le système immunitaire (les lymphocytes) a la capacité de reconnaître et de détruire des cellules anormales en voie de cancérisation, et protège ainsi l'individu de l'émergence d'un cancer. Ce concept, longtemps controversé, a connu une renaissance il y a une dizaine d'années par la démonstration formelle de son existence dans des modèles de souris déficientes pour des gènes ou des cellules impliqués dans la réponse immunitaire $[2-4,37](\rightarrow)$. Ces données s'intègrent actuellement

$\rightarrow$ Voir la Synthèse de W.H. Fridman et C. Sautès-Fridman, dans une théorie plus large dénom- page 359 de ce numéro mée immunoediting, dans laquelle le système immunitaire exerce une surveillance immunitaire, créant une pression de sélection qui aboutit à l'émergence de clones tumoraux résistant à l'attaque immune. La pertinence clinique de ces données expérimentales a été fournie par la mise en évidence d'antigènes de tumeurs

Cet article fait partie du numéro thématique publié par médecine/sciences en avril 2014 et intitulé « Microenvironnement tumoral ».

\section{Intérêt de l'immunoscore pour prédire l'évolution clinique}
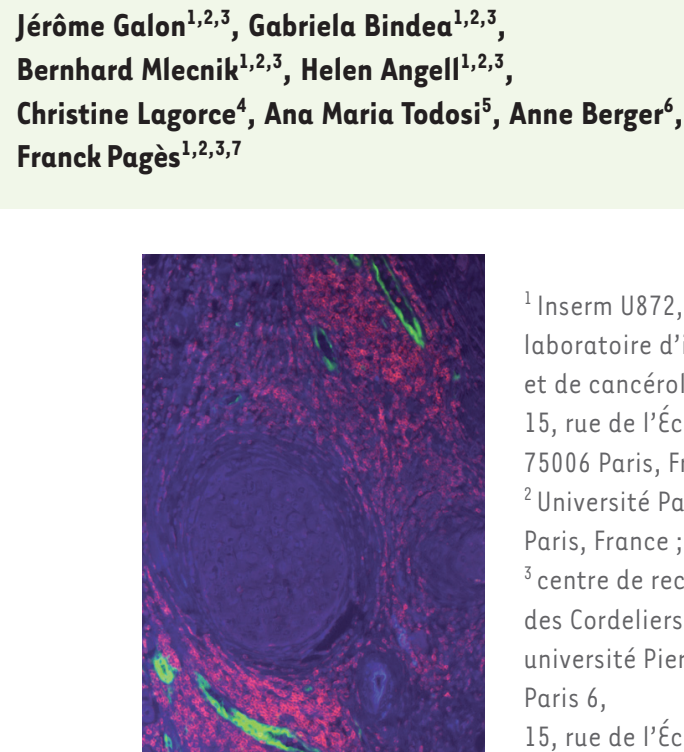

${ }^{1}$ Inserm U872,

laboratoire d'immunologie et de cancérologie intégratives, 15, rue de l'École de Médecine, 75006 Paris, France ;

${ }^{2}$ Université Paris Descartes,

Paris, France;

${ }^{3}$ centre de recherche des Cordeliers, université Pierre et Marie Curie Paris 6,

15, rue de l'École de Médecine, Paris, France

${ }^{4}$ hôpital Avicenne, département reconnus par les lymphocytes $T$ cytotoxiques [5], et par la démonstration d'un impact majeur de la qualité de l'infiltrat immunitaire et, particulièrement, des lymphocytes T cytotoxiques et mémoires, sur l'évolution tumorale [6-9]. Ainsi, se dessine une nouvelle prise en charge des patients prenant en compte l'extension tumorale et la qualité de la réaction immunitaire in situ, pour une optimisation du suivi et de la thérapie des patients à risque de récidive. De récents succès spectaculaires de l'immunothérapie antitumorale ont été obtenus en thérapie adoptive par transfert de lymphode pathologie, Bobigny, France ; ${ }^{5}$ département de chirurgie, université de médecine et pharmacie Gr T Popa lasi, Roumanie ;

${ }^{6}$ Assistance publique-hôpitaux de Paris, hôpital européen Georges Pompidou (HEGP), département de chirurgie générale et digestive, 20 , rue Leblanc, 75015 Paris, France ;

${ }^{7}$ Assistance publique-hôpitaux de Paris, HEGP,

département d'immunologie, 20 , rue Leblanc, 75015 Paris, France.

jerome.galon@crc.jussieu.fr cytes $T[10,11]$, par des vaccins antitumoraux, tels que le premier vaccin cellulaire Sipuleucel-T approuvé par la Food and drug administration (FDA) [12], et par des biothérapies utilisant des anticorps monoclonaux bloquant des molécules inhibitrices de la réponse lymphocytaire T, tels que les anti-CTLA-4 (cytotoxic T-lymphocyte antigen 4) (ipilimumab) [13, 38], ou les anti-PD-1 (programmed cell death 1)/PD-L1 (programmed cell death ligand 1) [1416]. Ces données cliniques récentes fournissent des éléments de preuve 
très forts attestant de la capacité de la composante immunitaire à contrôler le processus tumoral.

\section{Signatures immunitaires pronostiques dans différents types de cancers}

Un marqueur pronostique est un paramètre clinique ou biologique permettant de prédire l'évolution clinique d'un patient, guidant ainsi le clinicien dans le choix de la stratégie thérapeutique. Les marqueurs pronostiques en cancérologie sont constitués, d'une part, par la classification AJCC (American joint committee on cancer)/UICC (union for international cancer control) TNM, résumant l'extension tumorale dans l'organe primaire (stade $T$ ), les ganglions lymphatiques drainant la tumeur (stade $\mathrm{N}$ ) et les organes distants (stade M) et, d'autre part, par des paramètres biologiques sériques (marqueurs tumoraux) et moléculaires prenant en compte les désordres moléculaires de la cellule tumorale et les modifications du microenvironnement tumoral $[8,17,18]$. L'extension tumorale constitue le facteur pronostique le plus important utilisé en routine clinique pour les tumeurs solides, telles que les cancers colorectaux. Malgré la puissance pronostique de ce système de classification, le devenir clinique des patients est très variable pour un même stade de la classification [19]. L'information pronostique de la classification TNM est donc limitée et ne prédit pas, par ailleurs, la réponse au traitement qui sera institué.

Récemment, des progrès importants ont été réalisés dans la perception du pronostic par l'analyse de la composante immunitaire du microenvironnement tumoral (Figure 1). Les tumeurs solides humaines présentent un infiltrat polymorphe et variable d'un patient à l'autre, composé de macrophages, de cellules dendritiques (DC), de polymorphonucléaires, de cellules NK (natural killer) et de cellules de l'immunité adaptative, et des lymphocytes B et T. II a été montré dans de nombreuses tumeurs solides colorectales $[7,9,20]$, dans des mélanomes, et dans des cancers de l'ovaire, de la tête et le cou, de la vessie, du sein, du foie, de la prostate, de la vessie et du poumon [6], qu'une forte infiltration lymphocytaire était associée à une survie prolongée. La majorité des études ont révélé qu'une forte infiltration intratumorale en lymphocytes $\mathrm{TCD}^{+}, \mathrm{TCD}^{+}$cytotoxiques et en lymphocytes T CD45RO+ mémoires était corrélée à une survie sans récidive prolongée et un accroissement de la survie globale [7]. Cette infiltration immunitaire se localise dans la tumeur (CT) et le front d'invasion (IM). Ce profil d'infiltration immunitaire est associé à une orientation immunitaire des lymphocytes T auxiliaires CD4 ${ }^{+}$de type 1 (THl, Thelper 1). Les facteurs de transcription T-bet (TBX21), IRF-1 (interferon [IFN] regulation factor-1) et STAT-1 (signal transducer and activator of transcription-1) sont mis en jeu, aboutissant à la production locale d'interleukine (IL)-12, d'IL-2 et d'IFN- $\gamma$, à la présence de lymphocytes $\mathrm{T} C D 8^{+}$activés présentant des marqueurs de cytotoxicité (granzymes $[G Z M])$, perforine 1 [PRFl], granulysine [GNLY]). Ces lymphocytes expriment les récepteurs de chimiokines CXCR3 et CCR5, ainsi que des molécules d'adhésion (MADCAMI [mucosal vascular addressin cell adhesion molecule 1], ICAMI [intercellular adhesion molecule 1], VCAMI [vascular cell adhesion molecule 1]) pour leur permettre

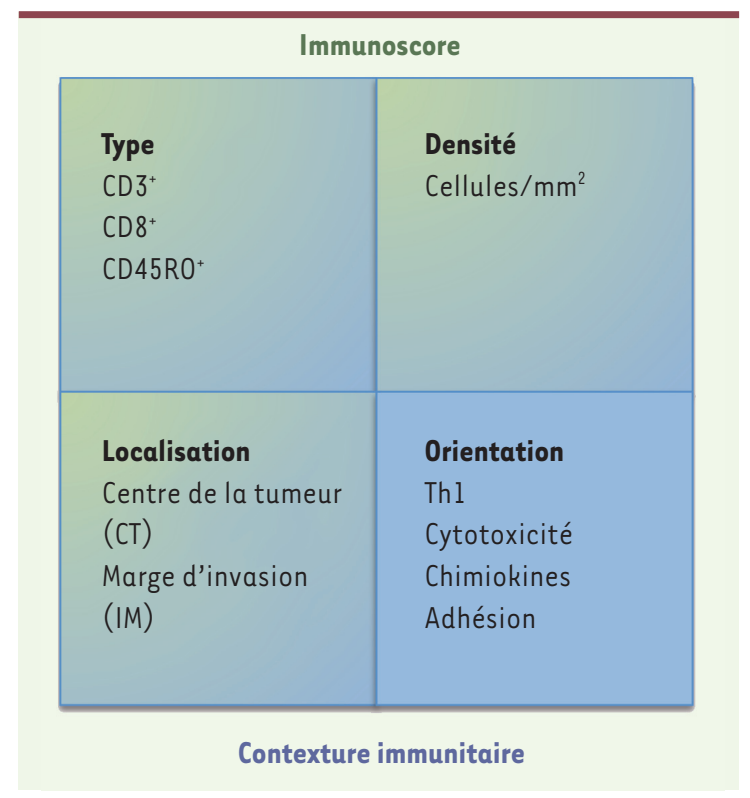

Figure 1. Représentation schématique de la contexture immunitaire et de l'immunoscore. La contexture immunitaire (en bleu) est composée de quatre éléments : type cellulaire, densité cellulaire, localisation dans la tumeur, et orientation fonctionnelle de la réponse immunitaire. L'immunoscore (en vert) est composé du type cellulaire, de la densité cellulaire, et de la localisation de ces cellules immunitaires dans la tumeur.

de se localiser sur le site tumoral riche en chimiokines (CCL5, CXCL9 et CXCL10, CX3CL1 et CCL2) (Figure 1). La chimio-attraction et l'adhésion semblent jouer un rôle crucial dans la détermination de la densité des cellules immunitaires intratumorales [21]. L'ensemble de ces données immunitaires, définissant un profil d'infiltration fortement associé au pronostic, ont été regroupées sous le terme de « contexture immunitaire » $[6-8,22$, 23] (Tableau I).

\section{L’immunoscore comme marqueur pronostique}

Les analyses rétrospectives de grandes cohortes de patients présentant un cancer ont démontré l'impact pronostique de l'évaluation de la composante immunitaire des tumeurs. Un test simple et puissant, appelé immunoscore, a été dérivé de la notion de contexture immunitaire pour une application en pratique clinique. L'immunoscore (I) est basé sur la détermination de la densité intratumorale de deux populations lymphocytaires $\left(\mathrm{CD}^{+} / \mathrm{CD} 45 \mathrm{RO}^{+}\right.$ou $\mathrm{CD}^{+} / \mathrm{CD}^{+}$ou $\left.\mathrm{CD} 8^{+} / \mathrm{CD} 45 \mathrm{RO}^{+}\right)$ dans la tumeur et son front d'invasion. Ceci est réalisé par une technique d'immunohistochimie sur coupe tissulaire couplée à un système d'analyse d'image. Les paramètres fournissent un système de quotation allant 


\begin{tabular}{|c|c|c|}
\hline Paramètres & $\begin{array}{l}\text { Contexture } \\
\text { immunitaire }\end{array}$ & Immunoscore \\
\hline Type & $\begin{array}{l}\mathrm{CTL}\left(\mathrm{CD}^{+}, \mathrm{CD} 8^{+}\right) \\
\text {Lymphocyte T mémoire } \\
\left(\mathrm{CD} 45 \mathrm{RO}^{+}\right)\end{array}$ & $\begin{array}{l}\mathrm{CTL}\left(\mathrm{CD}^{+}, \mathrm{CD} 8^{+}\right) \\
\text {Lymphocyte T mémoire } \\
\left(\mathrm{CD} 45 \mathrm{RO}^{+}\right)\end{array}$ \\
\hline Localisation & $\begin{array}{l}\text { Tumeur (CT), marge } \\
\text { d'invasion (IM) } \\
\text { Présence et qualité des } \\
\text { TLS }\end{array}$ & $\begin{array}{l}\text { Tumeur (CT), marge } \\
\text { d'invasion (IM) }\end{array}$ \\
\hline Densité & Connue (cellules $/ \mathrm{mm}^{2}$ ) & $\begin{array}{l}\text { Valeurs seuils prédéfinies } \\
\text { (cellules } / \mathrm{mm}^{2} \text { ) }\end{array}$ \\
\hline $\begin{array}{l}\text { Orientation } \\
\text { fonctionnelle }\end{array}$ & $\begin{array}{l}\text { Orientation Thl } \\
\text { cytotoxicité } \\
\text { chimiokines, adhésion, } \\
\text { cytokines }\end{array}$ & NA \\
\hline
\end{tabular}

Tableau I. Caractéristiques et paramètres associés à la contexture immunitaire et à l'immunoscore. NA : non associé ; TLS : tertiary lymphoid structures.

d'un immunoscore $0(10)$, caractérisé par une faible densité des deux types de cellules dans les deux régions, à un immunoscore 4 (14), pour des tumeurs présentant une forte densité des deux populations cellulaires dans les deux régions tumorales (Tableau I, Figure 2). La valeur pronostique de ce critère immunitaire a été démontrée pour l'ensemble des patients présentant un cancer colorectal, à tous les stades de la classification histopathologique (AJCC/UICC TNM). Dans les cancers de stade précoce localisés à la paroi digestive sans envahissement ganglionnaire ou à distance (AJCC/UICC TNM stades I-II) [24], l'immunoscore (10 à 14) subdivise les patients en sous-groupes présentant des différences importantes de survie sans récidive et de survie globale $(p<0,0001)$. Cinq ans après le diagnostic, seuls $4,8 \%$ des patients ayant des tumeurs avec des densités élevées en cellules $\mathrm{CD} 8^{+}$et $\mathrm{CD} 45 \mathrm{RO} 0^{+}$ont récidivé, et $86,2 \%$ ont survécu. En revanche, lorsque la densité de ces populations immunitaires dans la tumeur est faible, $75 \%$ des patients ont récidivé et seuls $27,5 \%$ ont survécu [24]. De la même manière, l'immunoscore a permis de subdiviser les patients présentant un cancer de stade avancé en des groupes de pronostic distinct et avec des différences majeures de survie [23].

De plus, il a été montré que l'immunoscore a une valeur pronostique supérieure à celle fournie par la classification TNM. Les analyses statistiques multivariées montrent que la valeur pronostique des critères de progression tumorale est statistiquement dépendante de celle de l'immunoscore. L'analyse combinée des deux paramètres révèle que l'immunoscore reste le seul critère significatif pour la survie sans récidive et la survie globale, alors que la classification AJCC/UICC TNM devient non significative [23]. Une partie de l'explication de la prépondérance statistique de l'immunoscore pourrait être que la densité de l'infiltrat $C D 8^{+}$est inversement corrélée avec le stade T dans les tumeurs qui ne vont pas récidiver, tandis que la densité en cellules T CD8 est faible, même au sein de cancers peu invasifs, dans le groupe de tumeurs qui vont récidiver [23].

Ainsi, l'intégration de l'immunoscore dans la classification des cancers pourrait se traduire par une amélioration de la précision du pronostic, mais aussi, potentiellement, de la prédiction de la réponse aux traitements $[25,26]$. Un bon marqueur pronostique doit être réalisable en routine clinique, simple, peu coûteux, rapide, robuste, reproductible, quantitatif, et puissant. L'immunoscore pourrait répondre à ces exigences [25]. De plus, d'autres marqueurs, en particulier immunitaires, pourraient être utilisés pour affiner la valeur pronostique de l'immunoscore.

\section{Autres marqueurs associés à la survie des patients}

À l'ère de la médecine personnalisée, de très nombreuses informations moléculaires sont maintenant accessibles. Des marqueurs pronostiques génétiques ont été proposés en cancérologie, mais seuls un petit nombre d'entre eux ont été intégrés en pratique clinique à visée pronostique.

Dans les cancers colorectaux, l'analyse de la génétique somatique tumorale a permis d'identifier trois groupes de tumeurs coliques: des tumeurs avec instabilité chromosomique, des tumeurs avec instabilité génétique et des tumeurs avec phénotype méthylateur. À chaque groupe de tumeurs correspondent des mutations distinctes. Ainsi, dans le premier groupe, on observe des mutations fréquentes du gène suppresseur de tumeur TP53, du gène APC (adenomatous polyposis coli), des oncogènes KRas et des mutations de la PI3 kinase (PI3KCA, phosphatidylinositol-4,5-biphosphate 3-kinase, catalytic subunit alpha). Dans les tumeurs avec instabilité génétique, le système de réparation des mésappariements de l'ADN est altéré, induisant des mutations fréquentes de gènes associés au cycle cellulaire ou à l'apoptose. Dans la pratique clinique, seul le statut d'instabilité génétique est recherché à visée pronostique. Ce test possède une utilité dans la définition du pronostic, mais aussi pour prédire la réponse à la chimiothérapie adjuvante à base de 5FU ( 5 fluoro-uracile) de ces tumeurs.

Les nouvelles techniques de séquençage à haut-débit ont mis en évidence que chaque type histologique de cancer possède en réalité une très forte hétérogénéité génomique [27]. De plus, chaque mutation transformante est le plus souvent associée à une combinatoire variable d'autres mutations de gènes clés qui ont un 


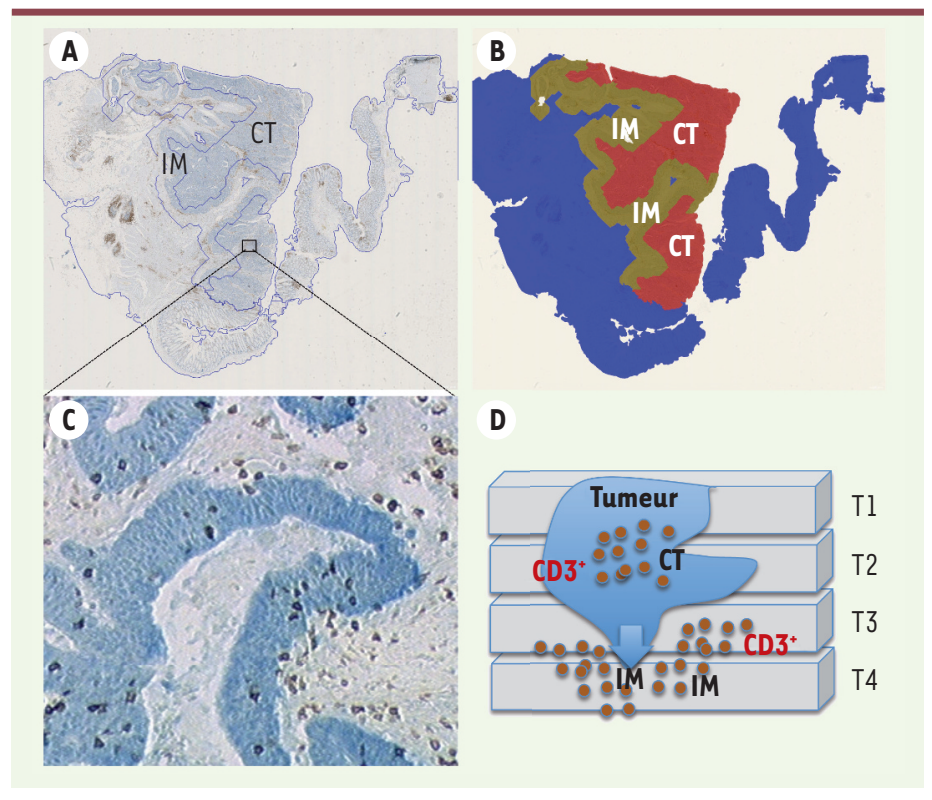

impact sur le comportement tumoral et son retentissement pronostique [28]. Ces données expliquent, en partie, la faible intégration de l'analyse génomique des tumeurs pour la prédiction de l'évolution tumorale.

Le développement des technologies à haut-débit, comme les puces à ADN, a permis d'analyser les profils d'expression géniques présents au site tumoral. Des signatures de gènes à visée pronostique ont été définies. Dans les cancers de l'ovaire, neuf signatures génétiques ont été rapportées. Cependant, presque aucun gène n'est commun aux différentes signatures. Cette situation se retrouve pour la plupart des signatures de gènes décrites dans d'autres types de cancers. Ainsi, la précision de ces prédicteurs reste encore insuffisante, et il est difficile d'identifier un biomarqueur pronostique robuste pour une analyse de routine.

\section{Marqueurs prédictifs de l'efficacité thérapeutique}

Les marqueurs pronostiques sont utiles pour évaluer le risque de récidive ou de décès d'un patient. Cependant, ils ne prédisent en rien la sensibilité de la tumeur à un traitement donné, un rôle qui concerne les biomarqueurs prédictifs de réponse [20]. Dans la pratique, il est fréquent que plusieurs options thérapeutiques soient possibles, telles que des chimiothérapies ou des thérapies ciblées. La présence d'un biomarqueur prédictif va permettre d'orienter la prescription médicale pour une meilleure efficacité thérapeutique et une moindre toxicité, dans un contexte de très forte inflation des coûts associés aux nouvelles thérapies.

À titre d'exemple, la surexpression de HER-2 (human epidermal growth factor [EGF] receptor-2) a été associée à une meilleure réponse à la doxorubicine dans le cancer du sein [29]. II a été montré que la présence d'une mutation activatrice dans une voie de signalisation empêche l'activité thérapeutique de biothérapies, inhibant l'interaction du récepteur et de son ligand. Ainsi, dans les cancers du côlon, la
Figure 2. Analyse morphologique et représentation schématique de l'extension tumorale. A. Pleine lame immunomarquée avec l'anticorps anti-CD3. B. Détection automatique du centre (cœur) de la tumeur (CT, rouge), de la marge d'invasion (IM, brun), et du tissu sain (violet). C. Grossissement d'une zone immunomarquée par $\mathrm{CD}^{+}$(marron) et d'une zome tumorale (bleu). D. Représentation schématique de l'extension tumorale (stades $\mathrm{Tl}, \mathrm{T} 2, \mathrm{~T} 3, \mathrm{~T} 4$ ). La tumeur est représentée en bleu et l'infiltrat immunitaire $\left(\mathrm{CD}^{+}\right)$en marron. $\mathrm{CT}$ : centre de la tumeur ; IM : marge d'invasion de la tumeur.

présence d'une mutation activatrice de KRas ou de la PIK3CA empêche l'activité antitumorale du Cetuximab, un anticorps anti-EGFR [30].

La composante immunitaire des cancers pourrait également jouer un rôle de biomarqueur prédictif de réponse. De récentes données ont démontré que les thérapies antitumorales conventionnelles, la radiothérapie et certaines drogues de chimiothérapie, telles que les anthracyclines et l'oxaliplatine, stimulent la réponse immunitaire antitumorale en induisant une mort cellulaire immunogène [31]. Il est par conséquent concevable que la nature de l'infiltration immunitaire au site tumoral (sa qualité, son intensité) puisse moduler la qualité de la réponse à ces thérapies. Le bénéfice de survie de patients atteints de CRC (colorectal cancer) ayant reçu une chimiothérapie à base de $5 \mathrm{FU}$ est grandement amélioré chez les patients qui présentent une forte infiltration de lymphocytes dans la tumeur (TIL) $(p=0,02)$ [32]. Étant donné que la présence de TIL reflète une réponse immunitaire adaptative, ces résultats suggèrent une interaction entre la réponse immunitaire et la chimiothérapie. Nous avons par ailleurs observé qu'une forte infiltration immunitaire intratumorale en lymphocytes $T$ cytotoxiques était prédictive d'une bonne réponse à la radiochimiothérapie dans les cancers du rectum (données non publiées). Notre équipe évalue actuellement l'impact de l'immunoscore pour prédire la réponse à ces thérapies.

Nous avons vu que de nouvelles approches thérapeutiques, telles que des agonistes des récepteurs Toll-like (TLR) $[33,39]$, des vaccinations à base de cellules dendritiques [34], des thérapies adoptives de lymphocytes T, et des biothérapies à base d'anticorps monoclonaux bloquant des signaux inhibiteurs de la réponse immunitaire, ont une activité antitumorale significative, avec des taux de guérison spectaculaires dans des essais randomisés de phase II et III. Ces essais cliniques modulant des molécules clés qui régulent l'activation lymphocytaire T (anti-CTLA-4 et anti-PD-1/anti-PDL-1) ont montré, dans des situations avancées de cancers métastatiques, 
que le système immunitaire n'est pas un système inerte, mais plutôt un système en état de suppression active que caractérise la coexistence d'une immunité antitumorale et de mécanismes immunosuppresseurs, reflétant probablement un échappement immunitaire partiel. Cependant, les réponses sont restreintes à une portion des patients et restent peu prévisibles. L'avènement de biomarqueurs prédictifs de réponse serait d'un grand intérêt clinique. Dans les essais cliniques utilisant des anticorps anti-CTLA-4 (ipilimumab), la surexpression de ID01 (indoleamine 2,3-dioxygénase 1, un immunosuppresseur classique) est corrélée à l'efficacité du traitement $[13,35]$. Dans le même cadre, il a été observé que la surexpression de FOXP3 (forkhead box P3, un marqueur de lymphocytes T régulateurs) et de la protéine IDO est corrélée positivement avec l'efficacité de l'ipilimumab [36]. De même, les tumeurs exprimant les ligands PD-1 semblent être sensibles à la thérapie anti-PD-1 [16]. La réalisation d'un immunoscore pour évaluer la qualité de l'infiltration immunitaire intratumorale avant traitement pourrait fournir un marqueur prédictif de réponse à ces thérapies immunomodulatrices.

\section{Conclusion}

La biologie des systèmes et les technologies à large échelle sont des approches puissantes pour découvrir les mécanismes associés à la progression tumorale et à la récidive chez les patients. En dépit d'un échappement partiel de la tumeur à la réponse immunitaire, la réaction immunitaire intratumorale est un paramètre majeur influençant la survie des patients. Les paramètres immunitaires les plus déterminants ont été regroupés selon un concept de «contexture » immunitaire qui prend en compte le type cellulaire, l'orientation fonctionnelle, la densité et la localisation des cellules immunitaires adaptatives dans différentes régions de la tumeur. Pour des évaluations immunitaires de routine, un test simple a été proposé, appelé immunoscore. Une validation internationale de l'immunoscore est actuellement en cours de réalisation afin d'intégrer ce nouveau paramètre à la classification TNM des cancers et aboutir à une classification de type TNM-I (TNM-immune) [25]. En outre, la contexture immunitaire et l'immunoscore pourraient permettre de prédire la réponse à de nombreuses thérapies [22]. Enfin, la connaissance des défauts de contexture immunitaire pourrait fournir de nouvelles stratégies thérapeutiques antitumorales. $\diamond$

\section{SUMMARY}

Intratumoral immune microenvironment and survival:

\section{the immunoscore}

The natural history of cancer involves interactions between the tumor and the host immune system. In humans, clinical and experimental data support the existence of a natural immune response against cancer. We provided evidence that the type, the density and the location of immune cells within the tumor strongly influence the prognosis, independently of the TNM classification. We established a methodology named "immunoscore" to assess in clinical practice the immune infiltrate. An international consortium of expert laboratories is currently testing the immunoscore in routine clinical settings for cancer classification. The availability of the mmunoscore could significantly improve the prognostic assessment of patients and better guide the therapeutic decision. This could result in the implementation of the immunoscore as a new component for the classification of cancer, designated TNM-I (TNMimmune). $\diamond$

\section{LIENS D'INTÉRÊT}

Les auteurs déclarent n'avoir aucun lien d'intérêt concernant les données publiées dans cet article.

\section{REMERCIEMENTS}

Ce travail a été réalisé par le laboratoire d'immunologie et de cancérologie intégratives, Inserm U872, et a reçu les soutiens de l'Institut national du cancer (INCa), du cancéropôle îlede-France, de l'Inserm, du Medlmmune, du Patar national research fund (national priorities research program award number NPRP09-1174-3-291), de the European commission (7FP, Geninca Consortium, grant 202230), du Cancer research for personalized medicine (CARPEM) et du LabEx Immunooncology.

\section{RÉFÉRENCES}

1. Burnet FM. The concept of immunological surveillance. Prog Exp Tumor Res $1970 ; 13: 1-27$.

2. Koebel CM, Vermi W, Swann JB, et al. Adaptive immunity maintains occult cancer in an equilibrium state. Nature 2007 ; 450 : 903-7.

3. Schreiber RD, Old LJ, Smyth MJ. Cancer immunoediting: integrating immunity's roles in cancer suppression and promotion. Science $2011 ; 331$ : 1565-70.

4. Shankaran V, Ikeda H, Bruce AT, et al. IFNgamma and lymphocytes prevent primary tumour development and shape tumour immunogenicity. Nature $2001 ; 410$ : 1107-11.

5. Van der Bruggen P, Traversari C, Chomez P, et al. A gene encoding an antigen recognized by cytolytic T lymphocytes on a human melanoma. Science 1991 ; $254: 1643-7$.

6. Fridman WH, Pages F, Sautes-Fridman C, Galon J. The immune contexture in human tumours: impact on clinical outcome. Nat Rev Cancer $2012 ; 12$ : 298-306.

7. Galon J, Costes A, Sanchez-Cabo F, et al. Type, density, and location of immune cells within human colorectal tumors predict clinical outcome. Science 2006 ; $313: 1960-4$

8. Galon J, Fridman WH, Pages F. The adaptive immunologic microenvironment in colorectal cancer: a novel perspective. Cancer Res 2007 ; 67 : 1883-6.

9. Pages F, Berger A, Camus M, et al. Effector memory T cells, early metastasis, and survival in colorectal cancer. $N$ Engl J Med 2005 ; $353: 2654-66$.

10. June CH. Adoptive T cell therapy for cancer in the clinic. J Clin Invest 2007 ; $117:$ 1466-76.

11. Rosenberg SA, Restifo NP, Yang JC, et al. Adoptive cell transfer: a clinical path to effective cancer immunotherapy. Nat Rev Cancer $2008 ; 8: 299$-308.

12. Kantoff PW, Higano CS, Shore ND, et al. Sipuleucel-T immunotherapy for castration-resistant prostate cancer. N Engl J Med 2010 ; 363 : 411-22.

13. Hodi FS, O'Day SJ, McDermott DF, et al. Improved survival with ipilimumab in patients with metastatic melanoma. N Engl J Med $2010 ; 363: 711-23$.

14. Brahmer JR, Tykodi SS, Chow LQ, et al. Safety and activity of anti-PD-LI antibody in patients with advanced cancer. N Engl J Med 2012 ; $366: 2455-65$.

15. Robert C, Thomas L, Bondarenko I, et al. Ipilimumab plus dacarbazine for previously untreated metastatic melanoma. N Engl J Med 2011 ; 364 : 2517-26.

16. Topalian SL, Hodi FS, Brahmer JR, et al. Safety, activity, and immune correlates of anti-PD-1 antibody in cancer. N Engl J Med 2012; 366 : 2443-54.

17. Bindea $G$, Mlecnik B, Fridman WH, et al. Natural immunity to cancer in humans. Curr Opin Immunol $2010 ; 22: 215-22$. 


\section{RÉFÉRENCES}

18. Ogino S, Galon J, Fuchs CS, Dranoff G. Cancer immunology - analysis of host and tumor factors for personalized medicine. Nat Rev Clin Oncol $2011 ; 8$ : 711-9.

19. Nagtegaal ID, Quirke P, Schmoll HJ. Has the new TNM classification for colorectal cancer improved care? Nat Rev Clin Oncol $2012 ; 9: 119-23$.

20. Angell HK, Galon J. From the immune contexture to the Immunoscore: the role of prognostic and predictive immune markers in cancer. Curr Opin Immunol $2013 ; 25: 261-7$.

21. Mlecnik B, Tosolini M, Charoentong P, et al. Biomolecular network reconstruction identifies T-cell homing factors associated with survival in colorectal cancer. Gastroenterology $2010 ; 138$ : $1429-40$.

22. Galon J, Angell HK, Bedognetti D, Marincola FM. The continuum of cancer immunosurveillance: prognostic, predictive and mechanistic signatures. Immunity 2013 ; 39 : 11-26.

23. Mlecnik B, Tosolini M, Kirilovsky A, et al. Histopathologic-based prognostic factors of colorectal cancers are associated with the state of the local immune reaction. J Clin Oncol 2011; 29:610-8.

24. Pages F, Kirilovsky A, Mlecnik B, et al. In situ cytotoxic and memory T cells predict outcome in patients with early-stage colorectal cancer. J Clin Oncol $2009 ; 27$ : 5944-51.

25. Galon J, Franck P, Marincola FM, et al. Cancer classification using the Immunoscore: a worldwide task force. J Transl Med $2012 ; 10: 205$.

26. Galon J, Pages F, Marincola FM, et al. The immune score as a new possible approach for the classification of cancer. J Transl Med 2012; $10: 205$.

27. Reis-Filho JS, Pusztai L. Gene expression profiling in breast cancer: classification, prognostication, and prediction. Lancet $2011 ; 378: 1812-23$.

28. Ding L, Getz G, Wheeler DA, et al. Somatic mutations affect key pathways in lung adenocarcinoma. Nature $2008 ; 455: 1069-75$.

29. Muss HB, Thor AD, Berry DA, et al. c-erbB-2 expression and response to adjuvant therapy in women with node-positive early breast cancer. $N$ Engl J Med 1994 ; 330 : 1260-6.

30. Lievre A, Bachet JB, Le Corre D, et al. KRAS mutation status is predictive of response to cetuximab therapy in colorectal cancer. Cancer Res $2006 ; 66: 3992-5$
31. Vacchelli $\varepsilon$, Galluzzi L, Fridman WH, et al. Trial watch: Chemotherapy with immunogenic cell death inducers. Oncoimmunology $2012 ; 1: 179-88$.

32. Morris $M$, Platell C, lacopetta B. Tumor-infiltrating lymphocytes and perforation in colon cancer predict positive response to 5 -fluorouracil chemotherapy. Clin Cancer Res 2008 ; 14 : 1413-7.

33. Vacchelli $\varepsilon$, Galluzzi L, Eggermont A, et al. Trial watch: FDA-approved Tolllike receptor agonists for cancer therapy. Oncoimmunology $2012 ; 1$ : 894-907.

34. Vacchelli $\varepsilon$, Martins I, Eggermont A, et al. Trial watch: Peptide vaccines in cancer therapy. Oncoimmunology $2012 ; 1: 1557-76$.

35. Schwartzentruber DJ, Lawson DH, Richards JM, et al. gpl00 peptide vaccine and interleukin-2 in patients with advanced melanoma. $N$ Engl J Med 2011 $364: 2119-27$.

36. Hamid 0 , Schmidt $H$, Nissan A, et al. A prospective phase II trial exploring the association between tumor microenvironment biomarkers and clinical activity of ipilimumab in advanced melanoma. J Transl Med 2011; $9: 204$.

37. Fridman WH, Sautès-Fridman C. Le microenvironnement tumoral : matrice nourricière, champ de bataille et cible thérapeutique des cancers. Med Sci (Paris) $2014 ; 30: 359-65$.

38. Robert C, Mateus C. Anticorps anti-CTLA-4 : une avancée thérapeutique majeure dans le traitement du mélanome métastatique. Med Sci (Paris) $2011 ; 27: 850-8$.

39. Garaude J. Mobiliser l'immunité innée dans le traitement des cancers. Med Sci (Paris) $2013 ; 29: 985-90$.

\section{STRESS, TRAUMATISMES ET INSOMNIES}

\section{de Jean-Pierre FRESCO}

TIRÉS À PART

J. Galon

\section{L'insomnie c'est}

\section{Français sur 5}

1 personne âgée sur 3

qui en souffrent

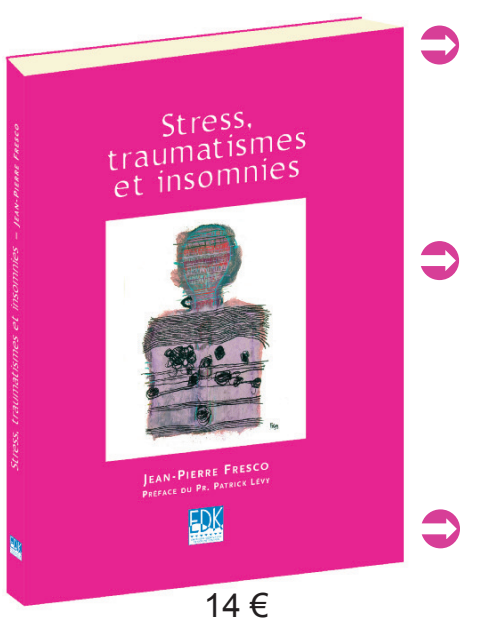

ISBN : 978-2-8425-4175-0

\section{Une étude}

sur les liens entre stress, traumatismes et insomnies.

\section{Une réflexion philosophique et sociétale sur les conditions de travail et de vie d'aujourd'hui.}

\section{Une analyse} d'un mal fréquent mais encore peu compris. 